RX J1015.5+0904:

\title{
A NEW POLAR AT THE LOWER PERIOD LIMIT
}

\author{
V. BURWITZ ${ }^{1}$, K. REINSCH ${ }^{1}$, A. D. SCHWOPE ${ }^{2}$, \\ K. BEUERMANN ${ }^{1,3}$, S. MENGEL ${ }^{2}$, P. NOTNI $^{2}$, \\ A. VAN TEESELING ${ }^{1}$, H.-C. THOMAS ${ }^{4}$ \\ 1. Universitäts-Sternwarte, Geismarlandstr. 11, D-37083 \\ Göttingen, Germany. (burwitz@usw051.dnet.gwdg.de) \\ 2. Astrophysikalisches Institut Potsdam, Germany \\ 3. MPI für Extraterrestrische Physik, Garching, Germany \\ 4. MPI für Astrophysik, Garching, Germany
}

\begin{abstract}
We present X-ray and optical observations of the ROSAT discovered polar (AM Her binary) RX J1015.5+0904 and first results of our analysis of the $\mathrm{X}$-ray and optical data. These results indicate a one-pole accretion geometry and an orbital period of $P=79.88 \mathrm{~m}$ for this $V \sim 17 \mathrm{mag}$ system.
\end{abstract}

\section{Introduction}

From our analysis of bright, soft, high-galactic-latitude ROSAT All-Sky Survey sources we extracted a set of AM Her candidates (Beuermann \& Thomas 1993; Beuermann \& Schwope 1994; Beuermann \& Burwitz 1995). Follow-up optical and X-ray observations led to the discovery of 27 new polars, more than doubling the pre-ROSAT sample. Here we present a summary of our detailed analysis of our X-ray (ROSAT survey, pointed PSPC and HRI) and optical (photometric and spectroscopic) observations of the new polar RX J1015.5+0904 from our sample of ROSAT discovered AM Her binaries.

\section{The ROSAT discovered polar RX J1015.5+0904}

The orbital period of the $V \sim 17$ mag optical counterpart of RX J1015.5 +0904 derived from all the X-ray and optical data is $P=79.88 \mathrm{~m}$, which is close to the lower limit for periods of cataclysmic variables. Our X-ray light curves indicate a one-pole accretion geometry with an absorption dip 
caused by the accretion stream crossing the line of sight (cf. Fig. 1). The optical light curve shows a double humped structure which is characteristic of cyclotron beaming. A complete analysis and more detailed discussion of our data will be presented in Burwitz et al. (1996).

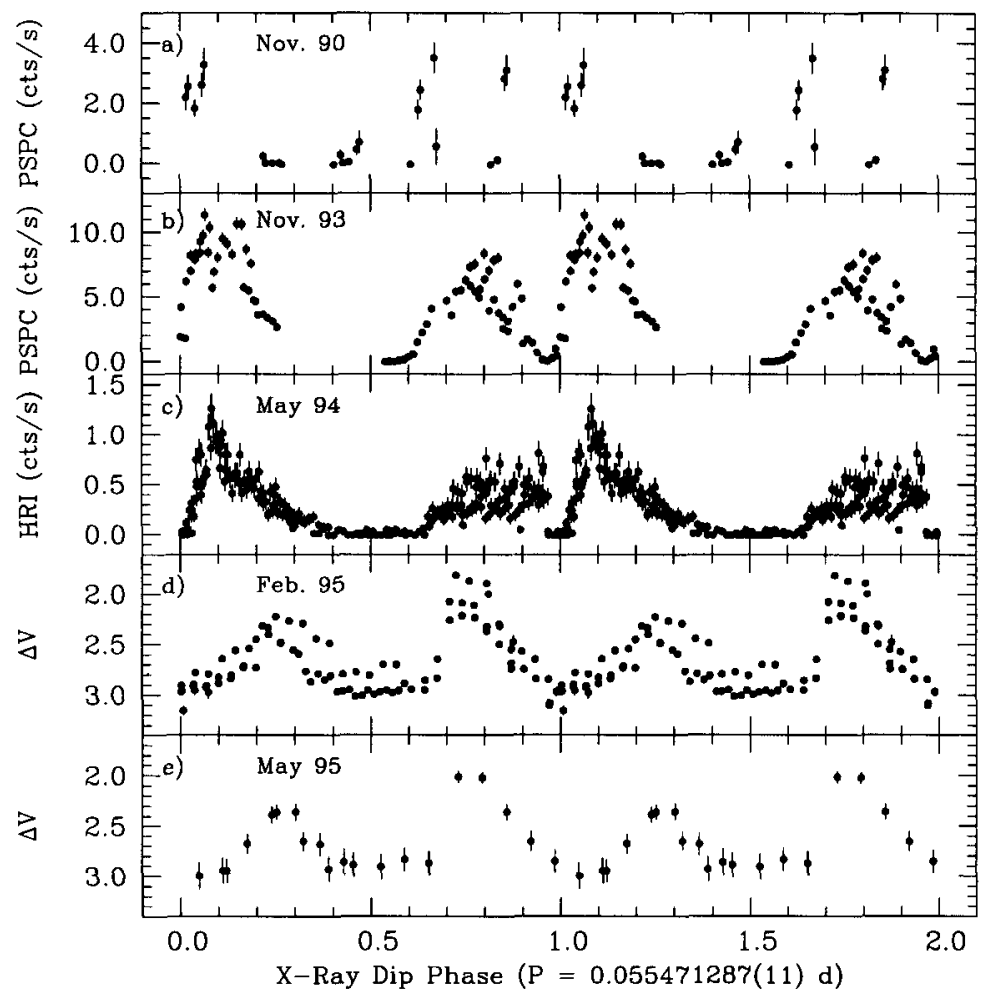

Figure 1. X-ray and optical light curves of RX J1015.5+0904. X-ray observations with ROSAT: (a) All-sky-survey (1990 November), (b) pointed PSPC observations (1993 November) and (c) pointed HRI observations (1994 May). Optical $V$ CCD photometry obtained with: (d) the Dutch $90 \mathrm{~cm}$ telescope at La Silla in 1995 February, (e) the 70 $\mathrm{cm}$ telescope in Potsdam in 1995 May. Phase zero is defined by the center of the X-ray dip in the HRI light curve: $T(\operatorname{dip})=$ HJD 2449488.35649(26) $+0.055471287(11)$ E.

\section{References}

Beuermann, K., Burwitz, V., 1995, in "Magnetic Cataclysmic Variables", eds D. Buckley, B. Warner, ASP Conf. Ser. 85, 99

Beuermann, K., Schwope, A.D., 1994, in "Interacting Binary Stars", ed. A. Shafter, ASP Conf. Ser. 56, 119

Beuermann, K., Thomas, H.-C., 1993, Adv. Space Res., 13(12), 115

Burwitz, V., Reinsch, K., Schwope, A.D., et al., 1996, A\&A, in prep 